\title{
Asymptotic Behavior of the Stochastic Rayleigh-van der Pol Equations with Jumps
}

\author{
ZaiTang Huang ${ }^{1}$ and ChunTao Chen ${ }^{2}$ \\ ${ }^{1}$ School of Mathematical Sciences, Guangxi Teachers Education University, Nanning 530001, China \\ ${ }^{2}$ College of Mathematics and Information Science, Guangxi University, Nanning 530004, China \\ Correspondence should be addressed to ZaiTang Huang; zaitanghuang@163.com
}

Received 20 May 2013; Revised 2 July 2013; Accepted 2 July 2013

Academic Editor: Józef Banaś

Copyright (c) 2013 Z. Huang and C. Chen. This is an open access article distributed under the Creative Commons Attribution License, which permits unrestricted use, distribution, and reproduction in any medium, provided the original work is properly cited.

We study the stability, attractors, and bifurcation of stochastic Rayleigh-van der Pol equations with jumps. We first established the stochastic stability and the large deviations results for the stochastic Rayleigh-van der Pol equations. We then examine the existence limit circle and obtain some new random attractors. We further establish stochastic bifurcation of random attractors. Interestingly, this shows the effect of the Poisson noise which can stabilize or unstabilize the system which is significantly different from the classical Brownian motion process.

\section{Introduction}

Gaussian process have been used to model many physical systems for quite a long time. However, researchers have increasingly been studying models from economics and from the natural sciences, where the underlying randomness contains jumps, which exhibit jump-like behavior. Such processes are being identified in the physical environment, in engineering systems, and in financial sectors. As it will quickly become apparent, the theory of RDS with jumps is nowhere near as well developed as the theory of continuous RDS, and in fact, is still in a relatively primitive stage. In particular, the theory of stochastic bifurcation is still in its infancy. Therefore, the field is both fertile and important [1$6]$.

A large class of phenomena can be described using stochastic differential equations drive white noise, in such distinct domains as economics, physics, or biology. Many analytical methods have been developed to characterize the solutions of such systems. In particular, the widely applied theory of stochastic bifurcations [7-10] allows a qualitative characterization of the asymptotic regimes of random dynamical systems. This theory is very handy to study nonlinear stochastic differential equations and is used to characterize the asymptotic behavior of complicated systems. Investigating the impact of Poisson noisy perturbations on such systems is hence of great interest and is currently an active field of research. In recent years, it has been shown in many different areas that applying Poisson noisy on a random system can lead to many counter-intuitive phenomena, such as Poisson noisy-induced stabilization [11]. From a mathematical perspective, understanding the interplays between white noise and Poisson noisy perturbations is a great challenge, with many applications. Several tools have been introduced, ranging from the theory of random dynamical systems $[5,6,12-14]$, the study of moment equations $[3,15]$. Unfortunately, no systematic method in the flavor of bifurcation theory for the analysis of the dynamics of nonlinear SDE drive Poisson noisy exists, and this is the central problem of the present paper.

We focus here on the dynamics of stochastic Rayleighvan der Pol equations with jumps. This type of equations may appear, for example, in diffusion approximations of physical models [16] or the classical model for a stock price [5]. The question we address is how the interplay between the stochastic jump system and diffusion system affects the behavior of the system. An important contribution of RDS theory to the field of stochastic bifurcations is characterized 
by a change in the shape of the invariant measure and stochastic Lyapunov exponent. We investigate the questions of stochastic stability and bifurcations, random attractors, and limits for stochastic Rayleigh-van der Pol equations with jumps, using combinations of appropriate tools such as Lyapunov direct method, Arnold asymptotic analysis approach, exponential martingale, and Lyapunov exponent.

The paper is organized of as follows. In Section 2, we give some preliminaries. We study the stochastic stability in Section 3. Random attractors are presented in Section 4. Random limits and stochastic bifurcation are further investigated in Section 5.

\section{Preliminaries}

In this paper, we analyze the behavior of the solutions of stochastic Rayleigh-van der Pol equations with jumps:

$$
\begin{gathered}
d x_{1}=x_{2} d t \\
d x_{2}=\left[\alpha x_{1}+\beta x_{2}-x_{2}^{3}-x_{1}^{2} x_{2}\right] d t \\
+\left[\sigma_{1} x_{1}+\sigma_{2} x_{2}+\sigma_{3}\right] \circ d W(t) \\
+\int_{Z}\left[\gamma_{1} c_{1}(z) x_{1}(t-)\right. \\
\left.\quad+\gamma_{2} c_{2}(z) x_{2}(t-)+\gamma_{3} c_{3}(z)\right] N(d t, d z),
\end{gathered}
$$

with initial condition $x(0)=x_{0}$ in $\mathfrak{R}^{2}$. The symbol denotes the Stratonovich calculus, $W(t)$ is a Brownian motion and $N(d t, d z)$ is the Poisson point process on $\mathfrak{R}_{+} \times Z$ with simultaneous jumps of probability zero with $\lambda(d z)$, the deterministic finite characteristic measure on a measurable space $Z$ such that $\widetilde{N}(d t, d z)=N(d t, d z)-\lambda(d z) d t$ is a $\mathscr{F}_{t^{-}}$ martingale measure. We also assume that $W(t)$ and $N(d t, d z)$ are independent. Let $\left(\Omega, \mathscr{F},\{\mathscr{F}\}_{t \geq 0} \mathbb{P}\right)$ be the underlying complete filtered probability space.

At the bifurcation points $\alpha=0$ and $\sigma_{3}=c_{3}(z)=0$, a linear change of variables yields

$$
\begin{aligned}
& d x(t) \\
& =\left(\begin{array}{ll}
0 & 0 \\
0 & \beta
\end{array}\right) x(t) d t \\
& +\left(\begin{array}{c}
a x_{2}^{3}(t)+b x_{2}^{2}(t) x_{1}(t)+c x_{2}(t) x_{1}^{2}(t)+d x_{1}^{3}(t) \\
-a x_{2}^{3}(t)-b x_{2}^{2}(t) x_{1}(t)-c x_{2}(t) x_{1}^{2}(t)-d x_{1}^{3}(t)
\end{array}\right) d t \\
& +\left(\begin{array}{ll}
\sigma_{11} & \sigma_{12} \\
\sigma_{21} & \sigma_{22}
\end{array}\right) x(t) \circ d W(t) \\
& +\int_{Z}\left(\begin{array}{ll}
\gamma_{11}(z) & \gamma_{12}(z) \\
\gamma_{21}(z) & \gamma_{22}(z)
\end{array}\right) x(t) N(d t, d z)
\end{aligned}
$$

If $Z\{-1,1\}$ and $N(d t, d z)$ are random telegraph process with $1 / \lambda$, the mean time between jumps, then stochastic equation (1) may be treated as informal derivative of the Poisson processes $N(t)$ with intensity $\lambda$ as

$$
\begin{gathered}
d x_{1}=x_{2} d t \\
d x_{2}=\left[\alpha x_{1}+\beta x_{2}-x_{2}^{3}-x_{1}^{2} x_{2}\right] d t \\
+\left[\sigma_{1} x_{1}+\sigma_{2} x_{2}+\sigma_{3}\right] d W(t) \\
+\left[\gamma_{1} x_{1}+\gamma_{1} x_{2}+\gamma_{1}\right] d N(t),
\end{gathered}
$$

where $\gamma_{i} \in \mathfrak{R}(i=1,2,3)$ are strength parameters and $N(t)$ is a Poisson process with intensity $\lambda$ over $(\Omega, \mathscr{F}, P)$.

\section{Stochastic Stability}

In the section, we would like to study the stability of the linearized stochastic Rayleigh-van der Pol equations driven by Poisson noise.

Theorem 1. Suppose $\sigma_{11}=\sigma_{22}=\sigma_{1}, \sigma_{12}=\sigma_{2} \neq 0, \sigma_{21}=-\sigma_{2}$ $\neq 0, \gamma_{12}=\gamma_{21}=0, \gamma_{11}=-\beta c(z)-1, \gamma_{22}=\beta c(z)-1$, if $\beta>0$, then the diffusion system corresponding to the linearized system (2) is asymptotically unstable with the Lyapunov exponent:

$$
\Lambda=\frac{\beta}{2}\left(1+\frac{I_{1}\left(\beta / 2 \sigma_{2}^{2}\right)}{I_{0}\left(\beta / 2 \sigma_{2}^{2}\right)}\right)>0
$$

However, the stochastic jump diffusion of the linearized system (2) is asymptotically stable if

$$
\frac{\beta}{2}\left(1+\frac{I_{1}\left(\beta / 2 \sigma_{2}^{2}\right)}{I_{0}\left(\beta / 2 \sigma_{2}^{2}\right)}\right)+\lambda(Z) \ln \beta<-\int_{Z} \ln |c(z)| \lambda(d z),
$$

where

$$
\begin{gathered}
I_{1}(x)=\frac{1}{2 \pi} \int_{0}^{2 \pi} e^{x \sin \varphi} d \varphi, \\
I_{0}(x)=\frac{1}{2 \pi} \int_{0}^{2 \pi} \sin \varphi e^{x \sin \varphi} d \varphi .
\end{gathered}
$$

Proof. We can rewrite the linearized system (2) as follows:

$$
\begin{aligned}
d x(t)= & \left(\begin{array}{ll}
0 & 0 \\
0 & \beta
\end{array}\right) x(t) d t+\left(\begin{array}{cc}
\sigma_{1} & \sigma_{2} \\
-\sigma_{2} & \sigma_{1}
\end{array}\right) x(t) \circ d W(t) \\
& +\int_{Z}\left(\begin{array}{cc}
-\beta c(z)-1 & 0 \\
0 & \beta c(z)-1
\end{array}\right) x(t) N(d t, d z),
\end{aligned}
$$

where $\sigma_{1} \neq 0, \sigma_{2} \neq 0$.

Denote by $\varphi$ the angle of $x \in \mathfrak{R}^{2}$. Then, $\varphi=\arctan$ $\left(x_{2} / x_{1}\right) \in[0,2 \pi)$, which depends on the quadrant of $\left(x_{1}, x_{2}\right) \in \mathfrak{R}^{2}$, and $\theta=(\cos \varphi, \sin \varphi) \in \mathbb{S}$. Let $\theta^{\perp}=$ $(\sin \varphi,-\cos \varphi)$. Then, $\theta^{\perp}$ is orthogonal to $\theta$. Thus, in terms of the new coordinates $(\rho, \varphi)$,

$$
x_{1}=e^{\rho} \cos \varphi, \quad x_{2}=e^{\rho} \sin \varphi .
$$


By Itô's formula, we have

$$
\begin{aligned}
d \rho(t)= & \beta \sin ^{3} \varphi(t) d t+\sigma_{1} d W(t) \\
& +\int_{Z} \ln (\beta|c(z)|) N(d t, d z), \\
d \varphi(t)= & \beta \sin \varphi(t) \cos \varphi(t) d t \\
& -\sigma_{2} d W(t) \int_{Z}[\pi(1-0.5 \operatorname{sgn}(c(z))) \\
& \left.-2 \varphi\left(t_{-}\right)\right] N(d t, d z) .
\end{aligned}
$$

Thus, the adjoint equation

$$
\begin{gathered}
\frac{1}{2} \frac{d^{2}}{d \varphi^{2}}\left(\sigma_{2}^{2} \psi\right)-\frac{d}{d \varphi}\left[\left(\frac{\beta}{2} \sin 2 \varphi\right) \psi\right] \\
+\int_{Z}\left[\psi\left(\pi(1-0.5 \operatorname{sgn}(c(z)))-\varphi\left(t_{-}\right)\right)\right. \\
-\psi(\varphi)] \lambda(d z)=0
\end{gathered}
$$

admits a unique periodic solution as the invariant probability density

$$
\psi(\varphi)=\psi_{0} \exp \left\{\frac{\beta}{2 \sigma_{2}} \sin 2 \varphi\right\},
$$

with

$$
\psi_{0}=\left(\int_{0}^{2 \pi} \exp \left\{\frac{\beta}{2 \sigma_{2}} \sin 2 \varphi\right\} d \varphi\right)^{-1}
$$

The Lyapunov exponent is as follows:

$$
\begin{aligned}
\Lambda & =\frac{\beta}{2}+\frac{\beta}{2} \int_{0}^{2 \pi} \sin 2 \varphi \psi(\varphi) d \varphi+\int_{Z} \ln (\beta|c(z)|) \lambda(d z) \\
& =\frac{\beta}{2}+\frac{\beta}{2} \frac{I_{1}\left(\beta / 2 \sigma_{2}^{2}\right)}{I_{0}\left(\beta / 2 \sigma_{2}^{2}\right)}+\int_{Z} \ln (\beta|c(z)|) \lambda(d z),
\end{aligned}
$$

where the integrals

$$
\begin{gathered}
I_{1}(x)=\frac{1}{2 \pi} \int_{0}^{2 \pi} e^{x \sin \varphi} d \varphi, \\
I_{0}(x)=\frac{1}{2 \pi} \int_{0}^{2 \pi} \sin \varphi e^{x \sin \varphi} d \varphi,
\end{gathered}
$$

are the modified Bessel functions which satisfy, respectively, the zero-order and the first-order Bessel equations of a purely imaginary argument:

$$
x^{2} \ddot{y}+x \dot{y}-x^{2} y=0, \quad x^{2} \ddot{y}+x \dot{y}-\left(x^{2}+1\right) y=0 .
$$

Note that $I_{0}(x)$ is even, while $I_{1}(x)$ is odd such that $I_{1}(x) / I_{0}(x)$ is between -1 and 1 , strictly increasing and at most linear growth. The diffusion system corresponding to (7) is asymptotically unstable with the Lyapunov exponent

$$
\Lambda_{0}=\frac{\beta}{2}\left(1+\frac{I_{1}\left(\beta / 2 \sigma_{2}^{2}\right)}{I_{0}\left(\beta / 2 \sigma_{2}^{2}\right)}\right)>0,
$$

if $\beta>0$. However, the stochastic jump diffusion of (7) is asymptotically stable if

$$
\begin{aligned}
\Lambda= & \frac{\beta}{2}\left(1+\frac{I_{1}\left(\beta / 2 \sigma_{2}^{2}\right)}{I_{0}\left(\beta / 2 \sigma_{2}^{2}\right)}\right) \\
& +\lambda(Z) \ln \beta+\int_{Z} \ln |c(z)| \lambda(d z)<0 .
\end{aligned}
$$

Remark. This shows the effect of Poisson noises which can stabilize the system in this respect. The alternative phenomenon $\Lambda>0$ also happens if the reverse inequality in (17) holds while $\beta<0$.

Theorem 2. Suppose the condition of Theorem 1 is satisfied and the stochastic jump-diffusion linearized system (2) is asymptotically stable, that is, $\Lambda<0$. Then,

$$
\begin{aligned}
& \mathbb{P}_{x_{0}}\left\{\sup _{s \geq t}|x(s)| \geq K\right\} \\
& \leq K(\Lambda, \sigma, c(z), \eta, \lambda)\left(\frac{\left|x_{0}\right|}{K}\right)^{-\Lambda / K(\Lambda, \sigma, c(z), \eta, \lambda)} \\
& \quad \times \exp \left(-\frac{\Lambda^{2} t}{2 K(\Lambda, \sigma, c(z), \eta, \lambda)}\right)
\end{aligned}
$$

Proof. Let $\mathscr{L}_{\theta}$ be the integro-differential operator corresponding to the jump-diffusion process $\theta(t)$ of (9), and let $f \in C^{2}(\mathbb{S})$. Then,

$$
\begin{aligned}
\mathscr{L}_{\theta} f(Y)= & \mathscr{H}_{0} f(Y)+\frac{1}{2} \sum_{i=1}^{m} \mathscr{H}_{i}^{2} f(Y) \\
& +\int_{Z}\{f(C(z) \star Y)-f(Y)\} \lambda(d z) .
\end{aligned}
$$

Since,

$$
\int_{\mathbb{S}}(-Q(\theta)+\Lambda) \psi(\theta) d \theta=0
$$

where $\psi$ is the unique invariant probability density of the jump-diffusion process of (9), then the integro-differential equation $\mathscr{L}_{\theta} v(\theta)=-Q(\theta)+\Lambda$ has a unique solution $v \in$ $C^{1}\left(\mathbb{S}^{d-1}\right)$. Then,

$$
\mathscr{L}\left\{\ln |x(t)|+v\left(\frac{x(t)}{|x(t)|}\right)\right\}=\Lambda<0
$$


Let $M(t)=M^{c}(t)+M^{d}(t)$ be a right continuous martingale defined as follows:

$$
\begin{gathered}
M^{c}(t)=\int_{0}^{t} \sum_{i=1}^{2}\left(\frac{x_{i}(t)}{|x(t)|^{2}}+\partial_{i} v(\theta(s)) B x(t)\right) d W(s), \\
M^{d}(t)=\int_{0}^{t} \int_{Z}\{\ln |\widetilde{C}(z) \theta(s-)|+v(\widetilde{C}(z) \star \theta(s-)) \\
-v(\theta(s-))\} \widetilde{N}(d s, d z),
\end{gathered}
$$

and define an operator $\star$ on $\mathbb{S}$ by $\widetilde{C}(z) \star \theta\left(s^{-}\right)=$ $\widetilde{C}(z) \theta(s-) /|\widetilde{C}(z) \theta(s-)|, \widetilde{C}(z)=I+C(z), \partial_{i} v=\partial v / \partial x_{i}$

$$
\begin{gathered}
B=\left(\begin{array}{cc}
\sigma_{1} & \sigma_{2} \\
-\sigma_{2} & \sigma_{1}
\end{array}\right) \\
C(z)=\left(\begin{array}{cc}
-\beta c(z)-1 & 0 \\
0 & \beta c(z)-1
\end{array}\right) .
\end{gathered}
$$

Note that $b_{i}(\cdot), i=1,2, \ldots, m$, and $c(\cdot, z)$ are $\mathscr{F}_{t}$-adapted processes. Let $M_{\mu, \nu}(t)=M_{\mu}^{c}+M_{\nu}^{d}(t)$ be a $\mathscr{F}_{t}$-local martingale such that

$$
\begin{gathered}
M_{\mu}^{c}(t)=\sum_{i=1}^{m} \mu_{i} \int_{0}^{t} b_{i}(s) d W(s), \\
M_{\nu}^{d}(t)=v \int_{0}^{t} \int_{Z} c(s, z) \widetilde{N}(d s, d z) d s,
\end{gathered}
$$

are, respectively, a continuous $\mathscr{F}_{t}$-local martingale and a pure jump $\mathscr{F}_{t}$-local martingale with

$$
\begin{gathered}
\left\langle M_{\mu}^{c}\right\rangle(t)=\sum_{i=1}^{m} \mu_{i}^{2} \int_{0}^{t} b_{i}^{2}(s) d W(s), \\
\left\lfloor M_{\nu}^{d}\right\rfloor(t)=\int_{0}^{t} \int_{Z}\left\{e^{\nu c(s, z)}-1-\nu c(s, z)\right\} \widetilde{N}(d s, d z) d s .
\end{gathered}
$$

Therefore, let $\kappa>0$; then the conditional quadratic variation of $M^{c}(t)$ is

$$
\begin{gathered}
\left\langle\kappa M^{c}\right\rangle(t) \\
=\kappa^{2} \sum_{i, j=1}^{2} \int_{0}^{t} \sigma_{i j}(x(s))\left(\frac{x_{i}(s)}{|x(s)|^{2}}+\partial_{i} v(\theta(s))\right) \\
\quad \times\left(\frac{x_{j}(t)}{|x(t)|^{2}}+\partial_{j} v(\theta(s))\right) d s
\end{gathered}
$$

$$
\begin{aligned}
=\kappa^{2} \sum_{i, j=1}^{2} \int_{0}^{t} \sigma_{i j}(x(s)) & \\
\times & \left\{\theta_{i}(s)+\sum_{k=1}^{2}\left[\delta_{i k}-\theta_{i}(s) \theta_{k}(s)\right] \frac{\partial v(\theta(s))}{\partial \theta_{k}}\right\} \\
\times & \left\{\theta_{i}(s)+\sum_{k=1}^{2}\left[\delta_{j k}-\theta_{j}(s) \theta_{k}(s)\right] \frac{\partial v(\theta(s))}{\partial \theta_{k}}\right\} d s \\
\leq \kappa^{2} K(B, v) t & \leq \kappa^{2} K(q, A, B, C) t .
\end{aligned}
$$

as $\sigma_{i j}(\theta)$ and $\partial v(\theta(s)) / \partial \theta_{k}$ are bounded on $\mathbb{S}$.

On the other hand,

$$
\begin{array}{r}
\left\lfloor\kappa M^{d}\right\rfloor(t)=\int_{0}^{t} \int_{Z}[\exp \{\kappa \ln |\widetilde{C}(z) \theta(s)| \\
+\kappa v(\widetilde{C}(z) \star \theta(s))-\alpha v(\theta(s))\}-1 \\
-\kappa \ln |\widetilde{C}(z) \theta(s)|-\kappa v(\widetilde{C}(z) \star \theta(s)) \\
+\kappa v(\theta(s))] \lambda(d z) d s \\
\leq \int_{0}^{t} \int_{Z}\left[\operatorname { e x p } \left\{\kappa \left(\ln \left|\beta^{2} c^{2}(z)\right| \vee\left|\beta^{2} c^{2}(z)-1\right|\right.\right.\right. \\
\quad+2\|v\|)\}-1 \\
\left.\quad-\kappa\left(\ln \left|\beta^{2} c^{2}(z)\right| \vee\left|\beta^{2} c^{2}(z)-1\right|\right)\right] \\
\quad \times \lambda(d z) d s \quad+2\|v\|)\}] \lambda(d z) \\
\leq \frac{\kappa^{2} t}{2} \int_{Z}\left[\operatorname { e x p } \left\{\kappa \left(\ln \left|\beta^{2} c^{2}(z)\right| \vee\left|\beta^{2} c^{2}(z)-1\right|\right.\right.\right. \\
\quad+\frac{\kappa^{2} t}{2} K(c, \beta, \lambda, v)=\frac{\alpha^{2} t}{2} K\left(\Lambda, c, \beta, \lambda, \sigma_{1}, \sigma_{2}\right) .
\end{array}
$$

Since

$$
\mathbb{E} \exp \left\{\left\langle\kappa M^{c}\right\rangle(t)\right\}<\infty, \quad \mathbb{E}\left\lfloor\kappa M^{d}\right\rfloor(t)<\infty,
$$

for all $t>0$, then

$$
\mathscr{E}(\kappa M) t=\exp \left\{\alpha M(t)-\frac{1}{2}\left\langle\kappa M^{c}\right\rangle(t)-\left\lfloor\kappa M^{d}\right\rfloor(t)\right\},
$$


is a $\mathscr{F}_{t}$ martingale. Denote by $(t)$ the decimal part of $t$. As

$$
\begin{aligned}
& \sup _{k \leq s-(t)<k+1} \kappa \ln |x(s)| \\
& \leq \sup _{k \leq s-(t)<k+1} \kappa\left\{\ln |x(s)|+v\left(\frac{x(s)}{|x(s)|}\right)\right. \\
& -\int_{0}^{s} \mathscr{L}\left[\ln |x(u)|+v\left(\frac{x(u)}{|x(u)|}\right)\right] d u \\
& +\sup _{k \leq s-(t)<k+1} \kappa\left\{-v\left(\frac{x(u)}{|x(u)|}\right)+\ln \left|x_{0}\right|\right. \\
& \left.+x_{0} \mid+v\left(\frac{x_{0}}{\left|x_{0}\right|}\right)\right\} \\
& \left.+\sup _{k \leq s-(t)<k+1}\left\{\kappa M(t)-\frac{x_{0}}{\left|x_{0}\right|}\right)+\Lambda s\right\} \\
& \left.+\frac{\kappa^{2}}{2} K\left(\Lambda, \beta, \delta_{1}, \sigma_{2}, \lambda\right)(k+(t)+1)-\left\lfloor\kappa M^{d}\right](t)\right\} \\
& +\kappa\left(2\|v\|+\ln \left|x_{0}\right|\right)+\kappa \Lambda(k+1),
\end{aligned}
$$

we have by the martingale inequality

$$
\begin{aligned}
& \mathbb{P}_{x_{0}}\left\{\sup _{s \geq t}|x(s)| \geq R\right\} \\
& \leq \sum_{k=[t]}^{\infty} \mathbb{P}_{x_{0}}\left\{\sup _{k \leq s-(t)<k+1} \kappa \ln |x(s)| \geq \kappa \ln R\right\} \\
& \leq \sum_{k=[t]}^{\infty} \mathbb{P}_{x_{0}} \\
& \times\left\{\sup _{k \leq s-(t)<k+1} \mathscr{E}(\kappa M)(s)\right. \\
& \geq \exp \left[\kappa\left(\ln R-\ln x_{0}-2\|v\|\right)\right. \\
& -\kappa \Lambda(k+(t))-\frac{\kappa^{2}}{2} K\left(\Lambda, \sigma_{1}, \sigma_{2}, \beta, c, \lambda\right) \\
& \times(k+(t)+1)]\} \\
& \leq \sum_{k=[t]}^{\infty}\left(\frac{\left|x_{0}\right|}{R}\right)^{\kappa} \\
& \times e^{\kappa K\left(\Lambda, \sigma_{1}, \sigma_{2}, \beta, c, \lambda\right) / 2+2 \kappa\|v\|+\left[\kappa \Lambda+\kappa^{2} K\left(\Lambda, \sigma_{1}, \sigma_{2}, \beta, c, \lambda\right) / 2\right](k+(t))} .
\end{aligned}
$$

In order to minimize the exponent in the inequality of (31), let

$$
\kappa=-\frac{\Lambda}{K\left(\Lambda, \sigma_{1}, \sigma_{2}, \beta, c, \lambda\right)}>0 .
$$

Then, we can obtain that

$$
\begin{aligned}
& \mathbb{P}_{x_{0}}\left\{\sup _{s \geq t}|x(s)| \geq R\right\} \\
& \leq\left(\frac{\left|x_{0}\right|}{R}\right)^{-\Lambda / K\left(\Lambda, \sigma_{1}, \sigma_{2}, \beta, c, \lambda\right)} \\
& \times e^{\left(\Lambda^{2} / 2-2 \Lambda\|v\|\right) / K\left(\Lambda, \sigma_{1}, \sigma_{2}, \beta, c, \lambda\right)} \\
& \times \sum_{k=[t]} e^{-\Lambda^{2}(k+(t)) / K\left(\Lambda, \sigma_{1}, \sigma_{2}, \beta, c, \lambda\right)} \\
&=\left(\frac{\left|x_{0}\right|}{R}\right)^{-\Lambda / K\left(\Lambda, \sigma_{1}, \sigma_{2}, \beta, c, \lambda\right)} \\
& \times \frac{\exp \left\{\left(\Lambda^{2} / 2-2 \Lambda\|v\|\right) / K\left(\Lambda, \sigma_{1}, \sigma_{2}, \beta, c, \lambda\right)\right\}}{1-\exp \left\{-\Lambda^{2} / 2 K\left(\Lambda, \sigma_{1}, \sigma_{2}, \beta, c, \lambda\right)\right\}} \\
& \times \exp \left\{-\frac{\Lambda^{2} t}{2 K\left(\Lambda, \sigma_{1}, \sigma_{2}, \beta, c, \lambda\right)}\right\} .
\end{aligned}
$$

As $\|v\|=\sup _{|\theta|=1}|v(\theta)|=K\left(\Lambda, \sigma_{1}, \sigma_{2}, \beta, c, \lambda\right)$, the result of Theorem 2 follows.

\section{Random Attractors}

In this section, we would like to study the random attractors of the stochastic Rayleigh-van der Pol equations driven by the Poisson noise.

Theorem 3. Equation (3) generates a smooth random dynamical system $(\varphi(t, \omega, x))$ with the Poisson processes which is global to the forward, that is, $D_{t}(\omega)=\mathfrak{R}^{2}$ for all $t>0$. Moreover, $\left(\varphi\left(t, \theta_{-t} \omega, x\right)\right)$ is $\mathscr{F}_{t}^{0}$ measurable.

Proof. We can rewrite system (3) as follows:

$$
\begin{gathered}
d x_{1}=x_{2} d t \\
d x_{2}=\left[\alpha x_{1}+\beta x_{2}-x_{2}^{3}-x_{1}^{2} x_{2}\right] d t \\
+\left[\sigma_{1} x_{1}+\sigma_{2} x_{2}+\sigma_{3}\right] d W(t) \\
+\left[\gamma_{1} x_{1}+\gamma_{1} x_{2}+\gamma_{1}\right] d N(t) .
\end{gathered}
$$

Choose a function

$$
V\left(x_{1}, x_{2}\right)=x_{1}^{2}+x_{2}^{2} \text {. }
$$


Applying Itô's formula to (35), we have

$$
\begin{aligned}
V\left(x_{1}, x_{2}\right)= & x_{10}^{2}+x_{20}^{2} \\
& +\int_{0}^{t}\left[2(1+\alpha) x_{1} x_{2}+\sigma_{1}^{2} x_{1}^{2}\right. \\
& \left.\quad+\left(2 \beta+\sigma_{2}^{2}\right) x_{2}^{2}+\sigma_{3}^{2}-x_{2}^{4}-x_{1}^{2} x_{2}^{2}\right] d s \\
& +2 \int_{0}^{t}\left[\sigma_{1} x_{1} x_{2}+\sigma_{2} x_{2}^{2}+\sigma_{3} x_{2}\right] d W(s) \\
& +\int_{0}^{t}\left[2 \gamma_{1} x_{1} x_{2}+\gamma_{1}^{2} x_{1}^{2}\right. \\
& \left.\quad+\left(2 \gamma_{2}+\gamma_{2}^{2}\right) x_{2}^{2}+2 \gamma_{3} x_{2}+\gamma_{3}^{2}\right] d N(s) .
\end{aligned}
$$

Using the Cauchy-Bunyakovsky-Schwarz inequality, then

$$
\begin{aligned}
2(1+ & \alpha) x_{1} x_{2}+\sigma_{1}^{2} x_{1}^{2}+\left(2 \beta+\sigma_{2}^{2}\right) x_{2}^{2} \\
\leq & {\left[|(1+\alpha)|+\sigma_{1}^{2}\right] x_{1}^{2}+\left[|(1+\alpha)|+\left|\left(2 \beta+\sigma_{2}^{2}\right)\right|\right] x_{2}^{2} } \\
& \times\left(\left[|1+\alpha|+\sigma_{1}^{2}\right] \vee\left[|1+\alpha|+\left|2 \beta+\sigma_{2}^{2}\right|\right]\right) V\left(x_{1}, x_{2}\right) \\
\sigma_{1} x_{1} x_{2}+\sigma_{2} x_{2}^{2}+\sigma_{3} x_{2} & \\
\leq & \frac{\left|\sigma_{1}\right|}{2} x_{1}^{2}+\left(\frac{\left|\sigma_{1}\right|}{2}+\left|\sigma_{2}\right|+\frac{\left|\sigma_{3}\right|}{2}\right) x_{2}^{2}+\frac{\left|\sigma_{3}\right|}{2} \\
\leq & \left(\frac{\left|\sigma_{1}\right|}{2}+\left|\sigma_{2}\right|+\frac{\left|\sigma_{3}\right|}{2}\right) V\left(x_{1}, x_{2}\right)+\frac{\left|\sigma_{3}\right|}{2} \\
2 \gamma_{1} x_{1} & x_{2}+\gamma_{1}^{2} x_{1}^{2}+\left(2 \gamma_{2}+\gamma_{2}^{2}\right) x_{2}^{2}+2 \gamma_{3} x_{2}+\gamma_{3}^{2} \\
\leq & \left(\left[\left|\gamma_{1}\right|+\gamma_{1}^{2}\right] \vee\left[2\left|\gamma_{2}\right|+\gamma_{2}^{2}+\left|\gamma_{3}\right|\right]\right) \\
& \times V\left(x_{1}, x_{2}\right)+\left|\gamma_{3}\right|+\gamma_{3}^{2} .
\end{aligned}
$$

From (36), we can obtain

$$
\begin{aligned}
& V\left(x_{1}, x_{2}\right) \\
& \leq V\left(t_{0}\right) \\
& +\int_{0}^{t}\left(\left[|(1+\alpha)|+\sigma_{1}^{2}\right] \vee\left[|(1+\alpha)|+\left|\left(2 \beta+\sigma_{2}^{2}\right)\right|\right]\right) \\
& \quad \times V\left(x_{1}, x_{2}\right) d s \\
& +2 \int_{0}^{t}\left[\left(\frac{\left|\sigma_{1}\right|}{2}+\left|\sigma_{2}\right|+\frac{\left|\sigma_{3}\right|}{2}\right) V\left(x_{1}, x_{2}\right)+\frac{\left|\sigma_{3}\right|}{2}\right] d W(s) \\
& +\int_{0}^{t}\left\{\left(\left[\left|\gamma_{1}\right|+\gamma_{1}^{2}\right] \vee\left[2\left|\gamma_{2}\right|+\gamma_{2}^{2}+\left|\gamma_{3}\right|\right]\right) V\left(x_{1}, x_{2}\right)\right. \\
& \left.\quad+\left|\gamma_{3}\right|+\gamma_{3}^{2}\right\} d N(s) .
\end{aligned}
$$

Suppose that $Y_{t}$ satisfies the following equation with jumps:

$$
\begin{aligned}
Y_{t}= & V\left(t_{0}\right) \\
& +\int_{0}^{t}\left(\left[|(1+\alpha)|+\sigma_{1}^{2}\right] \vee\left[|(1+\alpha)|+\left|\left(2 \beta+\sigma_{2}^{2}\right)\right|\right]\right) Y_{s} d s \\
& +2 \int_{0}^{t}\left[\left(\frac{\left|\sigma_{1}\right|}{2}+\left|\sigma_{2}\right|+\frac{\left|\sigma_{3}\right|}{2}\right) Y_{s}+\frac{\left|\sigma_{3}\right|}{2}\right] d W(s) \\
& +\int_{0}^{t}\left\{\left(\left[\left|\gamma_{1}\right|+\gamma_{1}^{2}\right] \vee\left[2\left|\gamma_{2}\right|+\gamma_{2}^{2}+\left|\gamma_{3}\right|\right]\right) Y_{s}\right. \\
& \left.\quad+\left|\gamma_{3}\right|+\gamma_{3}^{2}\right\} d N(s)
\end{aligned}
$$

then $Y_{t}$ is finite on any finite interval $[0, T]$ for any $T>0$. Hence, (38) and (39) yield that the process $V\left(x_{1}, x_{2}\right)$ is finite on $[0, T]$ for any $0<T<+\infty$; that is, the random dynamical system $(\varphi(t, \omega, x))$ is global to the forward.

Corollary 4. Suppose the condition of Theorem 3 is satisfied. Then the random dynamical system $(\varphi(t, \omega, x))$ generated by (3) has the following additional properties:

(i) $D_{t}\left(\theta_{-t}\right)=\mathfrak{R}^{2}$ for all $t \geq 0$;

(ii) $R_{t}\left(\theta_{-t}\right) \downarrow E(\omega)$ as $t \uparrow \infty$.

Proof. Since all $t \in \mathfrak{R}, \varphi(t, \omega): D_{t}(\omega) \rightarrow R_{t}(\omega)$ is (local) diffeomorphism of the random(open) domain $D_{t}(\omega) \subset R^{2}$ into the random range $R_{t}(\omega)=\varphi(t, \omega) D_{t}(\omega) \subset \Re^{2}$ where $D_{t}(\omega) \downarrow$ as $t \uparrow \infty$, and $\varphi(0, \omega)=I d_{\Re^{2}}, E(\omega)=\bigcap_{t \in \Re} D_{t}(\omega)=$ $\{x: \varphi(t, \omega, x)$ exists for all times $\} . D(\omega)$ is the set of all initial values $x$ whose orbits never explode. It may happen that $D(\omega)=\emptyset$.

For $t \geq 0$, Theorem 3 yields that $(\varphi(t, \omega, x))$ is nonexplosive; we have the property (i) by replacing $\omega$ with $\theta_{-t} \omega$. Theorem 3 implies that $R_{t}\left(\theta_{-t} \omega\right)=\mathfrak{R}^{2}$ for $t \leq 0$ and $R_{t}\left(\theta_{t} \omega\right) \subset R_{s}\left(\theta_{s} \omega\right)$ for $0 \leq s \leq t$, respectively. Since $R_{t}\left(\theta_{-t} \omega\right)=D_{-t}(\omega)$, the definition of $E(\omega)$ yields that (ii) is true.

Lemma 5. Suppose $k>-1$. If $a-c^{2} / 2+\lambda \log (1+k)<0$, then the stochastic differential equation with the Poisson processes

$$
\begin{gathered}
d X_{t}=\left(a X_{t}+b\right) d t+\left(c X_{t}+h\right) d W(t)+\left(k X_{t}+f\right) d N(t), \\
X_{0}=x
\end{gathered}
$$

has the unique invariant measure which is a Dirac measure supported by

$$
\begin{aligned}
\xi(t)=[c & \int_{-\infty}^{0} e^{-\left(a-c^{2} / 2\right) t-c W_{s}-L N_{t}} d s \\
& +h \int_{-\infty}^{0} e^{-\left(a-c^{2} / 2\right) t-c W_{s}-L N_{t}} d W(s) \\
& \left.+f \int_{-\infty}^{0} e^{\left.-\left(a-c^{2} / 2\right) t-c W_{s}-L N_{t}\right)} d N_{s}\right],
\end{aligned}
$$


and $\xi(t)$ attracts all points with exponential speed, where $L=$ $\log (1+k)$ and $\lambda$ is the intensity of a Poisson process $N$ on $\mathfrak{R}$.

Proof. By Itô's formula, we obtain

$$
\begin{aligned}
& \varphi(t, \omega) x \\
& =e^{\left(a-c^{2} / 2\right) t+c W_{s}+L N_{t}[x}+c \int_{0}^{t} e^{-\left(a-c^{2} / 2\right) t-c W_{s}-L N_{t}} d s \\
& +h \int_{0}^{t} e^{-\left(a-c^{2} / 2\right) t-c W_{s}-L N_{t}} d W(s) \\
& \left.+f \int_{0}^{t} e^{\left.-\left(a-c^{2} / 2\right) t-c W_{s}-L N_{t}\right)} d N_{s}\right],
\end{aligned}
$$

is the random dynamical system generated by (40). Since $\lim _{t \rightarrow-\infty} N_{t}(\lambda t)=1$ and $a-c^{2} / 2+\lambda L<0$, we have the following integrals:

$$
\begin{gathered}
\int_{-\infty}^{0} e^{-\left(a-c^{2} / 2\right) t-c W_{s}-L N_{t}} d s<\infty, \\
\int_{-\infty}^{0} e^{-\left(a-c^{2} / 2\right) t-c W_{s}-L N_{t}} d W(s)<\infty, \\
\int_{-\infty}^{0} e^{-\left(a-c^{2} / 2\right) t-c W_{s}-L N_{t}} d N_{s}<\infty .
\end{gathered}
$$

Since (40) is affine with stable linear part, then, (42) and Theorem 5.6.1 of Arnold in [7] imply that the unique invariant measure of (40) is the Dirac measure supported by

$$
\begin{aligned}
\xi(t)= & \lim _{t \rightarrow \infty} \varphi(-t, \omega)^{-1} x \\
= & {\left[c \int_{-\infty}^{0} e^{-\left(a-c^{2} / 2\right) s-c W_{s}-L N_{s}} d s\right.} \\
& +h \int_{-\infty}^{0} e^{-\left(a-c^{2} / 2\right) s-c W_{s}-L N_{s}} d W(s) \\
& \left.+f \int_{-\infty}^{0} e^{\left.-\left(a-c^{2} / 2\right) s-c W_{s}-L N_{s}\right)} d N_{s}\right],
\end{aligned}
$$

for all $x \in \mathfrak{R}$ and $\xi(t)$ attracts all points with exponential speed.

Theorem 6. Suppose the condition of Theorem 3 is satisfied and the coefficient in (3) satisfies $\gamma_{1}+\gamma_{2}>-1, \sigma_{3}=\gamma_{3}=0$ and

$$
(1+\alpha+\beta)+2 \lambda \log \left[1+\left(\gamma_{1}+\gamma_{2}\right)\right]<\frac{\left(\sigma_{1}+\sigma_{2}\right)^{2}}{2}
$$

Then the random dynamical system generated by (3) possesses the unique parameter-dependent tempered random attractor $A$ with domain of attractor $\mathscr{D}(A)$ containing the universe of sets Cl( $\mathcal{U})$, generated by

$$
\mathcal{U}=\left\{(D(\omega))_{\omega \in \Omega}: D(\omega) \subset \mathfrak{R}^{2} \text { is a tempered random set }\right\} .
$$

Moreover, the random attractor $A$ is measurable with respect to $\mathscr{F}^{0}{ }_{-\infty}=\sigma\left\{N_{t}, t \leq 0\right\}$.

Proof. Choose a function

$$
V\left(x_{1}, x_{2}\right)=x_{1}^{2}+2 x_{1} x_{2}+x_{2}^{2}
$$

Applying Itô's formula, we have

$$
\begin{gathered}
V\left(x_{1}, x_{2}\right) \\
=V\left(t_{0}\right)+\int_{0}^{t}\left[2(1+\alpha+\beta) x_{1} x_{2}\right. \\
+\left(2 \alpha+\sigma_{1}^{2}\right) x_{1}^{2}+\left(2 \beta+\sigma_{2}^{2}+2\right) x_{2}^{2} \\
\left.-x_{2}^{4}-2 x_{1} x_{2}^{3}-x_{1}^{3} x_{2}-x_{1}^{2} x_{2}^{2}\right] d s \\
+2 \int_{0}^{t}\left[\sigma_{1} x_{1}^{2}+\left(\sigma_{1}+\sigma_{2}\right) x_{1} x_{2}+\sigma_{2} x_{2}^{2}\right] d W(s) \\
+\int_{0}^{t}\left[2\left(\gamma_{1}+\gamma_{2}\right) x_{1} x_{2}+\left(2 \gamma_{1}+\gamma_{1}^{2}\right) x_{1}^{2}\right. \\
\left.+\left(2 \gamma_{2}+\gamma_{2}^{2}\right) x_{2}^{2}\right] d N(s) .
\end{gathered}
$$

This implies

$$
\begin{aligned}
& V\left(x_{1}, x_{2}\right) \\
& =V\left(t_{0}\right)+\int_{0}^{t}\left[(1+\alpha+\beta) V\left(x_{1}, x_{2}\right)+A\left(x_{1}, x_{2}\right)\right] d s \\
& \quad+\int_{0}^{t}\left[\left(\sigma_{1}+\sigma_{2}\right) V\left(x_{1}, x_{2}\right)+B\left(x_{1}, x_{2}\right)\right] d W(s) \\
& \quad+\int_{0}^{t}\left[\left(\gamma_{1}+\gamma_{2}\right) V\left(x_{1}, x_{2}\right)+C\left(x_{1}, x_{2}\right)\right] d N(s),
\end{aligned}
$$

where

$$
\begin{gathered}
A\left(x_{1}, x_{2}\right)=\left(\alpha+\sigma_{1}^{2}-1-\beta\right) x_{1}^{2}+\left(\beta+\sigma_{2}^{2}+1-\alpha\right) x_{2}^{2} \\
-x_{2}^{4}-2 x_{1} x_{2}^{3}-x_{1}^{3} x_{2}-x_{1}^{2} x_{2}^{2}, \\
B\left(x_{1}, x_{2}\right)=\left(\sigma_{1}-\sigma_{2}\right)\left(x_{1}-x_{2}\right), \\
C\left(x_{1}, x_{2}\right)=\left(\gamma_{1}+\gamma_{1}^{2}-\gamma_{2}\right) x_{1}^{2}+\left(\gamma_{2}+\gamma_{2}^{2}-\gamma_{1}\right) x_{2}^{2} .
\end{gathered}
$$

Note that $A\left(x_{1}, x_{2}\right) \leq K\left(\alpha, \beta, \sigma_{1}, \sigma_{2}, \gamma_{1}, \gamma_{2}\right)=: K, B\left(x_{1}, x_{2}\right) \leq$ $K\left(\alpha, \beta, \sigma_{1}, \sigma_{2}, \gamma_{1}, \gamma_{2}\right), C\left(x_{1}, x_{2}\right) \leq K\left(\alpha, \beta, \sigma_{1}, \sigma_{2}, \gamma_{1}, \gamma_{2}\right)$, where $K$ is a positive constant independent of $x_{1}$ and $x_{2}$.

From (49), we have

$$
\begin{aligned}
V\left(x_{1}, x_{2}\right) \leq & V\left(t_{0}\right)+\int_{0}^{t}\left[(1+\alpha+\beta) V\left(x_{1}, x_{2}\right)+K\right] d s \\
& +\int_{0}^{t}\left[\left(\sigma_{1}+\sigma_{2}\right) V\left(x_{1}, x_{2}\right)+K\right] d W(s) \\
& +\int_{0}^{t}\left[\left(\gamma_{1}+\gamma_{2}\right) V\left(x_{1}, x_{2}\right)+K\right] d N(s) .
\end{aligned}
$$


Denote $\varphi\left(t, \omega,\left(x_{1}, x_{2}\right)\right)$ and $\psi\left(t, \omega,\left(x_{1}, x_{2}\right)\right)$; then random dynamical systems are generated by (3) and

$$
\begin{aligned}
Z_{t} \leq & V\left(t_{0}\right)+\int_{0}^{t}\left[(1+\alpha+\beta) Z_{s}+K\right] d s \\
& +\int_{0}^{t}\left[\left(\sigma_{1}+\sigma_{2}\right) Z_{s}+K\right] d W(s) \\
& +\int_{0}^{t}\left[\left(\gamma_{1}+\gamma_{2}\right) Z_{s}+K\right] d N(s),
\end{aligned}
$$

respectively. The random dynamical systems generated by (52) are

$$
\begin{aligned}
& \psi(t, \omega) x \\
& =e^{\left[(1+\alpha+\beta)-\left(\sigma_{1}+\sigma_{2}\right)^{2} / 2\right] t+\left(\sigma_{1}+\sigma_{2}\right) W_{t}+\log \left(1+\gamma_{1}+\gamma_{2}\right) N_{t}} \\
& \quad \times\left[x+K \int_{0}^{t} e^{-\left[(1+\alpha+\beta)-\left(\sigma_{1}+\sigma_{2}\right)^{2} / 2\right] s-\left(\sigma_{1}+\sigma_{2}\right) W_{s}-\log \left(1+\gamma_{1}+\gamma_{2}\right) N_{s}} d s\right. \\
& \quad+K \\
& \quad \times \int_{0}^{t} e^{-\left[(1+\alpha+\beta)-\left(\sigma_{1}+\sigma_{2}\right)^{2} / 2\right] s-\left(\sigma_{1}+\sigma_{2}\right) W_{s}-\log \left(1+\gamma_{1}+\gamma_{2}\right) N_{s}} d W(s) \\
& \quad+K \\
& \left.\quad \times \int_{0}^{t} e^{\left[-(1+\alpha+\beta)-\left(\sigma_{1}+\sigma_{2}\right)^{2} / 2\right] s-\left(\sigma_{1}+\sigma_{2}\right) W_{s}-\log \left(1+\gamma_{1}+\gamma_{2}\right) N_{s}} d N_{s}\right] .
\end{aligned}
$$

Thus, from (51), we have

$$
V\left(\varphi(t, \omega),\left(x_{1}, x_{2}\right)\right) \leq V\left(\psi(t, \omega),\left(x_{1}, x_{2}\right)\right) .
$$

By Lemma 5 and (45), we obtain that $\psi(t, \omega)$ has the unique invariant measure which is a Dirac measure supported by

$$
\begin{aligned}
& r(\omega) \\
& =K e^{\left[(1+\alpha+\beta)-\left(\sigma_{1}+\sigma_{2}\right)^{2} / 2\right] t+\left(\sigma_{1}+\sigma_{2}\right) W_{t}+\log \left(1+\gamma_{1}+\gamma_{2}\right) N_{t}} \\
& \quad \times\left\{\int_{0}^{t} e^{-\left[(1+\alpha+\beta)-\left(\sigma_{1}+\sigma_{2}\right)^{2} / 2\right] s-\left(\sigma_{1}+\sigma_{2}\right) W_{s}-\log \left(1+\gamma_{1}+\gamma_{2}\right) N_{s}} d W(s)\right. \\
& \left.\quad+\int_{0}^{t} e^{\left[-(1+\alpha+\beta)-\left(\sigma_{1}+\sigma_{2}\right)^{2} / 2\right] s-\left(\sigma_{1}+\sigma_{2}\right) W_{s}-\log \left(1+\gamma_{1}+\gamma_{2}\right) N_{s}} d N_{s}\right\}
\end{aligned}
$$$$
>0 \text {. }
$$

It is easy to prove that $\psi\left(t, \theta_{-t} \omega\right) x\left(\theta_{-t} \omega\right) \rightarrow r(\omega)$ as $t \rightarrow \infty$ for any initial value $x(\omega) \in \mathfrak{R}_{+}$such that $e^{\left[-(1+\alpha+\beta)+\left(\sigma_{1}+\sigma_{2}\right)^{2} / 2\right] t} x\left(\theta_{-t} \omega\right) \rightarrow 0$ for some $a>0$. Hence, we may the universe of set

$$
\overline{\mathscr{U}}=\left\{I(\omega) \subset \mathfrak{R}_{+} \text {is a tempered random set }\right\} ;
$$

that is, the random variable $\eta(\omega)=\sup _{x \in I(\omega)} x$ satisfies $\lim _{t \rightarrow \infty} \log ^{+}\left[\eta\left(\theta_{t} \omega\right) / t\right]=0$ for any $I=\overline{\mathscr{U}}$. From the results of
Arnold in [7, Proposition 4.17], it yields $\lim _{t \rightarrow \infty} e^{-\varepsilon t} \eta\left(\theta_{-t} \omega\right)=$ 0 for any $\varepsilon>0$.

It is clear that $\overline{\mathscr{U}}$ is closed under inclusion and $\Omega \times\{x\} \in \overline{\mathscr{U}}$ for all $x \in \mathfrak{R}^{+}$.

Next, we will prove that the random set $[0,(1+\varepsilon) r]$ is forward invariant and absorbing for $\psi(t, \omega)$ with respect to the universe $\overline{\mathcal{U}}$ for any $\varepsilon>0$.

In fact, the absorbing property follows from the definition of $\overline{\mathcal{U}}$ and Schenk-Hoppé [9, Theorem 4.1]. We only prove that $\psi(t, \omega)[0,(1+\varepsilon) r(\omega)] \subset\left[0,(1+\varepsilon) r\left(\theta_{t} \omega\right)\right]$ for all $t \geq 0$. Since $\psi(t, \omega)$ is a nonnegative function on $\Re$ and $\psi(t, \omega) x<$ $\psi(t, \omega) y$ for $x<y$, it suffices to show $\psi(t, \omega)(1+\varepsilon) r(\omega) \leq$ $(1+\varepsilon) r\left(\theta_{t} \omega\right)$, it is equivalent that prove

$$
\psi\left(t, \theta_{-t} \omega\right)(1+\varepsilon) r\left(\theta_{-t} \omega\right) \leq(1+\varepsilon) r(\omega) .
$$

From (53), one can obtain

$$
\begin{aligned}
& \psi\left(t, \theta_{-t} \omega\right)(1+\varepsilon) r\left(\theta_{-t} \omega\right) \\
& =r(\omega) \\
& \quad+\varepsilon K \\
& \quad \times\left\{\int_{-\infty}^{-t} e^{-\left[(1+\alpha+\beta)-\left(\sigma_{1}+\sigma_{2}\right)^{2} / 2\right] s-\left(\sigma_{1}+\sigma_{2}\right) W_{s}-\log \left(1+\gamma_{1}+\gamma_{2}\right) N_{s}} d W(s)\right. \\
& \left.\quad+\int_{-\infty}^{-t} e^{\left[-(1+\alpha+\beta)-\left(\sigma_{1}+\sigma_{2}\right)^{2} / 2\right] s-\left(\sigma_{1}+\sigma_{2}\right) W_{s}-\log \left(1+\gamma_{1}+\gamma_{2}\right) N_{s}} d N_{s}\right\} .
\end{aligned}
$$

It implies that (57) is true.

Since

$$
\begin{aligned}
& r\left(\theta_{-t} \omega\right) \\
& =K e^{-\left[(1+\alpha+\beta)-\left(\sigma_{1}+\sigma_{2}\right)^{2} / 2\right] t+\left(\sigma_{1}+\sigma_{2}\right) W_{-t}+\log \left(1+\gamma_{1}+\gamma_{2}\right) N_{-t}} \\
& \quad \times\left[\int_{-\infty}^{-t} e^{-\left[(1+\alpha+\beta)-\left(\sigma_{1}+\sigma_{2}\right)^{2} / 2\right] s-\left(\sigma_{1}+\sigma_{2}\right) W_{s}-\log \left(1+\gamma_{1}+\gamma_{2}\right) N_{s}} d W(s)\right. \\
& \left.\quad+\int_{-\infty}^{-t} e^{\left[-(1+\alpha+\beta)-\left(\sigma_{1}+\sigma_{2}\right)^{2} / 2\right] s-\left(\sigma_{1}+\sigma_{2}\right) W_{s}-\log \left(1+\gamma_{1}+\gamma_{2}\right) N_{s}} d N_{s}\right]
\end{aligned}
$$$$
\leq r(\omega)
$$

one has

$$
e^{-\epsilon t} r\left(\theta_{-t} \omega\right) \longrightarrow 0, \quad t \longrightarrow \infty,
$$

for any $\epsilon>0$. It means that $[0,(1+\varepsilon) r(\omega)] \in \overline{\mathcal{U}}$ for any $\varepsilon>0$.

For any $\varepsilon>0$, define the subset $B(\omega)$ of $\mathfrak{R}^{2}$ by

$$
B(\omega)=V^{-1}([0,(1+\varepsilon)] r(\omega)) .
$$

This is a nonempty compact set by the surjectivity and continuity of $V(x, y)$ and the fact that preimages of bounded sets are bounded under $V(x, y)$. One can prove that the random set $B(\omega)$ satisfies the assumptions of Schenk-Hoppé [9, Theorm 4.1]. 
The measurability of $B(\omega)$ may be implied by Lemma 5.7 of Schenk-Hoppé [9]. Using the surjectivity and nonnegativity of $V$ and (54), one has

$$
\begin{aligned}
& \psi\left(t, \theta_{-t} \omega\right)\left[0,(1+\varepsilon) r\left(\theta_{-t} \omega\right)\right] \subset[0,(1+\varepsilon) r(\omega)] \\
& \Longleftrightarrow \psi\left(t, \theta_{-t} \omega\right) V\left(B\left(\theta_{-t} \omega\right)\right) \subset V(B(\omega)) \\
& \Longrightarrow V\left(\psi\left(t, \theta_{-t} \omega\right) B\left(\theta_{-t} \omega\right)\right) \subset V(B(\omega)),
\end{aligned}
$$

for all $t \geq 0$. It implies

$$
\begin{aligned}
\psi\left(t, \theta_{-t} \omega\right) B\left(\theta_{-t} \omega\right) & \subset V^{-1}\left(V\left(\psi\left(t, \theta_{-t} \omega\right) B\left(\theta_{-t} \omega\right)\right)\right) \\
& \subset V^{-1}(V(B(\omega))=B(\omega)
\end{aligned}
$$

that is, $B$ is forward invariant.

For any $D \in \mathcal{U}$, we have that the random variable $\eta(\omega)=$ $\sup \left\{\|(x, y)\|^{2}:(x, y) \in D(\omega)\right\}$ grows subexponentially fast by the definition of $\mathcal{U} . V(x, y) \leq 3\left(x^{2}+y^{2}\right)+5 \leq 3\|(x, y)\|^{2}+5$ yields the random variable $\sup \{x \in V(D(\omega))\} \leq \eta(\omega)+5$ which grows sub-exponentially fast; that is, $V(D) \in \overline{\mathcal{U}}$.

As the proof of (62) and (63), we have that there is a $t(\omega, D)>0$ such that, for all $t>t(\omega, D)$,

$$
\begin{aligned}
& V\left(\varphi\left(t, \theta_{-t} \omega\right) D\left(\theta_{-t} \omega\right)\right) \\
& \subset \psi\left(t, \theta_{-t} \omega\right) V\left(D\left(\theta_{-t} \omega\right)\right) \subset[0,(1+\varepsilon) r(\omega)]=V(B(\omega)),
\end{aligned}
$$

by $(59)$ and the set $[0,(1+\varepsilon) r(\omega)]$ absorbing any set in $\overline{\mathcal{U}}$. It shows the absorption of any set in $\mathcal{U}$.

Finally, we prove the existence of a neighborhood of $B$ in $\mathcal{U}$. In fact, for any $B \in \mathcal{U}$, Proposition 3.2(iii) of SchenkHoppé [9] implies that there exists a random variable $\rho(\omega)>$ 0 such that $B(\omega) \subset S(\rho(\omega))=\left\{x \in \mathfrak{R}^{2},\|x\| \rho(\omega)\right\}$. It is clear that $S(b \rho(\omega)) \in \mathcal{U}$ and that $S(\rho(\omega))$ is a neighborhood of $B$ for all $b>1$.

By Theorem 4.1 of Schenk-Hoppé [9], we finish the proof. Because of $r(\omega) \in \mathscr{F}_{-\infty}^{0}$, we have that the attractor $A$ is $\mathscr{F}_{-\infty}^{0}$ measurable.

\section{Stochastic Bifurcation}

In this section, we would like to study the random limit circle and stochastic bifurcation of the stochastic Rayleigh-van der Pol equations driven by the Poisson noise.

Theorem 7. Assume that $\sigma_{3}=\gamma_{1}=\gamma_{2}=\gamma_{3}=0$. Then the Rayleigh-van der Pol equations (3) undergoes Hopf bifurcation; thus the stochastic system (3) occurs a random limits cycle.

Proof

Step 1. Let $c_{1}(z)=c_{2}(z)=c_{3}(z)=\sigma_{3}=\gamma_{1}=\gamma_{2}=\gamma_{3}=0$; then (3) becomes

$$
\begin{aligned}
d x= & \left(\begin{array}{cc}
0 & 1 \\
\alpha-\frac{\sigma_{1}}{2} & \beta-\frac{\sigma_{2}}{2}
\end{array}\right) x d t \\
& +\left(\begin{array}{c}
0 \\
-x_{1}^{2} x_{2}-x_{2}^{3}
\end{array}\right) d t+\left(\begin{array}{cc}
0 & 0 \\
\sigma_{1} & \sigma_{2}
\end{array}\right) x \circ d W(t) .
\end{aligned}
$$

The linearized RDS $\mathrm{D} \varphi$ is generated by the linearization of (65), namely, the white noise case by

$$
\begin{aligned}
\dot{v}= & \left(\begin{array}{cc}
0 & 1 \\
\alpha-\frac{\sigma_{1}}{2} & \beta-\frac{\sigma_{2}}{2}
\end{array}\right) v \\
& +\left(\begin{array}{cc}
0 & 0 \\
-2 x_{1} x_{2} & -3 x_{2}^{2}
\end{array}\right) v+\left(\begin{array}{cc}
0 & 0 \\
\sigma_{1} & \sigma_{2}
\end{array}\right) v \circ \dot{W}(t) .
\end{aligned}
$$

For any invariant measure $\nu$, the trace formula gives $\lambda_{1}(\nu)+$ $\lambda_{2}(\nu)=\beta-\sigma_{2} / 2-3 \gamma \mathbb{E}_{\nu} x_{1}^{2}$. In particular, for $\nu=\delta_{0}$, we obtain $\lambda_{1}(\nu)+\lambda_{2}(\nu)=\beta-\sigma_{2} / 2$.

We also need the eigenvalues of the linearization of the deterministic system $\sigma_{1}=\sigma_{2}=0$ at $x=0$,

$$
\dot{v}=\left(\begin{array}{cc}
0 & 1 \\
\alpha-\frac{\sigma_{1}}{2} & \beta-\frac{\sigma_{2}}{2}
\end{array}\right) v
$$

which are

$$
\lambda_{1,2}=\frac{\beta-\sigma_{2} / 2}{2} \pm \sqrt{\frac{\left(\beta-\sigma_{2} / 2\right)^{2}}{4}+\alpha-\frac{\sigma_{1}}{2}} .
$$

For $\left(\beta-\sigma_{2} / 2\right)^{2} / 4+\alpha-\sigma_{1} / 2>0$, we have two real eigenvalues, while for $\left(\beta-\sigma_{2} / 2\right)^{2} / 4+\alpha-\sigma_{1} / 2<0$, we have a pair of complex-conjugate eigenvalues:

$$
\lambda_{1,2}=\frac{\beta-\sigma_{2} / 2}{2} \pm i \omega_{d}, \quad \omega_{d}:=\sqrt{-\frac{\left(\beta-\sigma_{2} / 2\right)^{2}}{4}-\alpha+\frac{\sigma_{1}}{2}},
$$

where $\omega_{d}$ represents the "damped eigenfrequency" of the linear system:

$$
\ddot{X}-\beta \dot{X}-\alpha X=0 .
$$

It is a general observation that noise splits deterministic multiplicities of eigenvalues. For $\beta^{2}<-4 \alpha$ (which we assume) and $\sigma_{1}=\sigma_{2}=0$, the deterministic linear system has two complex-conjugate eigenvalues $\beta / 2 \pm \omega_{d}$, which amounts to just one Lyapunov exponent $\lambda_{1}(0, \beta)=\beta / 2$ with multiplicity 2. For $\sigma_{1} \neq 0$ and $\sigma_{2} \neq 0$, however, the linearized SDE

$$
\dot{v}=\left(\begin{array}{cc}
0 & 1 \\
\alpha-\frac{\sigma_{1}}{2} & \beta-\frac{\sigma_{2}}{2}
\end{array}\right) v+\left(\begin{array}{cc}
0 & 0 \\
\sigma_{1} & \sigma_{2}
\end{array}\right) v \circ \dot{W}(t),
$$

has two different simple Lyapunov exponents $\lambda_{i}(\mu, \alpha$, $\left.\sigma_{1}, \sigma_{2}\right)(i=1,2)$ which satisfy $\lambda_{1}\left(\alpha, \beta, \sigma_{1}, \sigma_{2}\right)+\lambda_{2}(\alpha, \beta$, $\left.\sigma_{1}, \sigma_{2}\right)=\beta-\sigma_{2} / 2$. For small $\sigma_{1}, \sigma_{2}$, we can use the asymptotic expansion given by Arnold [7, Theorem 9.4.11] and others; namely, when $\sigma_{1} \rightarrow 0, \sigma_{2} \rightarrow 0$, then the Lyapunov exponent of (71) is as follows:

$$
\lambda_{1,2}=\frac{\beta-\sigma_{2} / 2}{2} \pm \frac{\sigma_{1}^{2}+\sigma_{2}^{2}}{8 \omega_{d}^{2}} S\left(2 \omega_{d}\right)+O\left(\max \left\{\sigma_{1}^{4}+\sigma_{2}^{4}\right\}\right)
$$


where $S(\cdot)$ is the spectral density of $\dot{W}(t)$, for the white noise case where $S(u) \equiv 1$. Furthermore, the rotation number expands as

$$
\rho(\mu, \alpha)=\omega_{d}-\frac{c\left(\sigma_{1}^{2}+\sigma_{2}^{2}\right)}{8 \omega_{d}^{2}} T\left(2 \omega_{d}\right)+O\left(\max \left\{\sigma_{1}^{4}+\sigma_{2}^{4}\right\}\right),
$$

where $T(\cdot)$ is the sine spectral density of $\dot{W}(t), c>0$.

Consequently, at the deterministic Hopf bifurcation point $\beta=0$, we have for the white noise case, neglecting $O\left(\max \left\{\sigma_{1}^{4}+\sigma_{2}^{4}\right\}\right)$ term,

$$
\begin{aligned}
\lambda_{2}\left(\sigma_{1}, \sigma_{2}, 0\right) & =-\frac{\sigma_{1}^{2}+\sigma_{2}^{2}}{8 \omega_{d}^{2}} S\left(2 \omega_{d}\right) \\
& <0<\lambda_{1}\left(\sigma_{1}, \sigma_{2}, 0\right)=\frac{\sigma_{1}^{2}+\sigma_{2}^{2}}{8 \omega_{d}^{2}} S\left(2 \omega_{d}\right)
\end{aligned}
$$

that is, at $\beta=\sigma_{2} / 2$, the top exponent has already cross 0 and is positive. Then the Rayleigh-van der Pol equation (3) undergoesed the Hopf bifurcation.

Step 2. It follows from (72) that the top Lyapunov exponent changes sign at $\beta_{D_{1}}=\left(\left(\sigma_{1}^{2}+\sigma_{2}^{2}\right) / 4 \alpha\right) S\left(2 \omega_{d}\right)+O\left(\max \left\{\sigma_{1}^{4}+\right.\right.$ $\left.\left.\sigma_{2}^{4}\right\}\right)<0$ and the second Lyapunov exponent changes sign at $\beta_{D_{2}}=-\left(\left(\sigma_{1}^{2}+\sigma_{2}^{2}\right) / 4 \alpha\right) S\left(2 \omega_{d}\right)+O\left(\max \left\{\sigma_{1}^{4}+\sigma_{2}^{4}\right\}\right)>0$.

For $\beta-\sigma_{2} / 2<\beta_{D_{1}}, \delta_{0}$ is stable, it is the unique invariant measure, and $A_{\beta}=\{0\}$ is the attractor of the $\operatorname{RDS} \varphi_{\beta}$ in $\mathbb{R}^{2}$.

We have a first bifurcation from $\delta_{0}$ at $\beta_{D_{1}}=\beta-$ $\sigma_{2} / 2<0$ of a stable ergodic measure $\nu^{1}\left(\sigma_{1}, \sigma_{2}, \beta\right)_{\omega}=$ $(1 / 2)\left(\delta_{x^{1}(\omega)}+\delta_{-x^{1}(\omega)}\right)$, which is a convex combination of two Dirac measures, sitting on the two "boundary points" of the one-dimensional unstable manifold of $x=0$, which is a saddle point. This situation persists for $\beta-\sigma_{2} / 2 \in\left(\beta_{D_{1}}, \beta_{D_{2}}\right)$. As shown before, $\beta_{P}=0 \in\left(\beta_{D_{1}}, \beta_{D_{2}}\right)$, $v^{1}$ undergoes a $\mathrm{P}$ bifurcation, and $\delta_{0}$ and $\nu^{1}$ are both Markov measures. Hence, $\rho=\mathbb{E} v^{1}$ solves the Fokker-Planck equation. The attractor $A_{\alpha}$ in (the universe of tempered sets of) $\mathbb{R}^{2}$ is the closure of the unstable manifold of $x=0$, the two "boundary points" supporting the measure $\nu^{1}$. In the punctured plane $\mathbb{R}^{2} \backslash\{0\}$, the attractor $A_{\beta}^{0}$ (in the universe of simply connected tempered sets) consists of the two-point set supp $v^{1}$.

At $\beta-\sigma_{2} / 2=\beta_{D_{2}}$, we have a second bifurcation from $\delta_{0}$ of a measure $v^{2}\left(\sigma_{1}, \sigma_{2}, \beta\right)_{\omega}=(1 / 2)\left(\delta_{x^{2}(\omega)}+\delta_{-x^{2}(\omega)}\right)$, which is again a convex combination of two Dirac measures. $\nu^{2}$ is a saddle point that has positive and negative Lyapunov exponents, while $\delta_{0}$ has two positive exponents.

For $\beta-\sigma_{2} / 2>\beta_{D_{2}}$, the stable measure $v^{1}$ is supported by the "boundary" of the unstable manifold of $\nu^{2}$. The closure of this unstable manifold is an invariant "circle" around $x=0$ (random limit cycle) and supports both measures $v^{1}$ and $v^{2}$. On this "circle," we have hyperbolic dynamics ( $\nu^{1}$ is attracting and $v^{2}$ is repelling), in contrast to the deterministic case, where the dynamics on the limit cycle is just rotation and the invariant measure is unique. The interior of the "circle" is the two-dimensional unstable manifold of 0 . Its closure is the attractor $A_{\beta}$ in $\mathbb{R}^{2}$. It carries all three invariant measures. In the punctured plane $\mathbb{R}^{2} \backslash\{0\}$, however, the attractor $A_{\beta}^{0}$ is the invariant "circle," in which we have two invariant measures, in particular, the unique Markov measure $v^{1}$. Thus, the Rayleigh-van der Pol equation (3) occurs a random limit cycle.

Proposition 8. Suppose the condition of Theorem 3 is satisfied and the coefficient in (3) is satisfied. Denote $A(\omega)$ the random attractor of the random dynamical systems $\varphi$ generated by the stochastic Rayleigh-van der Pol equation (3). Then,

(i) the random attractor $A$ supports all invariant measures;

(ii) the unstable set of any invariant measure $\mu$ is contained in random attractor; that is, $M_{\mu}^{+}(\omega) \subset A(\omega)$;

(iii) there exists an invariant Markov measure $\mu$ supported by $\partial A(\omega)$; that this, $\mu_{\omega}$ is $\mathscr{F}_{-\infty}^{0}$ measurable.

Proof. It is the same as the proof in Proposition 7.5 of SchenkHoppé [9].

Proposition 9. Suppose the condition of Theorem 3 is satisfied and the coefficient in (3) is satisfied and $\alpha=-1, \sigma_{1} \geq 0, \sigma_{2} \geq$ $0, \gamma_{1}>\gamma_{2} \geq 0$. For $-2<\beta<2$, we can obtain that the Lyapunov exponents $\Lambda_{1}$ and $\Lambda_{2}$ of the linear stochastic Rayleigh-van der Pol equations (3)

$$
\begin{gathered}
d x_{1}=x_{2} d t \\
d x_{2}=\left[-x_{1}+\beta x_{2}\right] d t+\left[\sigma_{1} x_{1}+\sigma_{2} x_{2}\right] d W(t) \\
+\left[\gamma_{1} x_{1}+\gamma_{2} x_{2}\right] d N(t),
\end{gathered}
$$

are bounded by

$$
\begin{aligned}
{[\beta+} & \left.\frac{\max \left\{\sigma_{1}^{2}, \sigma_{2}^{2}\right\}}{\left.1+\frac{|\beta|}{2}\right]}\right] \\
& -\frac{\left[\left(\left(2 \sigma_{2}+\sigma_{1}\right)+|\beta|\left(\sigma_{1}+\sigma_{2} / 2\right)\right) /(1+|\beta| / 2)\right]^{2}}{2} \\
& +\lambda \log \left(1+\frac{2+\gamma_{1}^{2}+\gamma_{1}|\beta|+\gamma_{2}^{2}+2 \gamma_{2}+|\beta| \gamma_{2} / 2}{1+|\beta| / 2}\right) \\
\leq & \Lambda_{2} \leq \Lambda_{1} \leq\left[\beta+\frac{\max \left\{\sigma_{1}^{2}, \sigma_{2}^{2}\right\}}{1-|\beta| / 2}\right] \\
& -\frac{\left[\left(\left(2 \sigma_{2}+\sigma_{1}\right)+|\beta|\left(\sigma_{1}+\sigma_{2} / 2\right)\right) /(1-|\beta| / 2)\right]^{2}}{2} \\
& +\lambda \log \left(1+\frac{2+\gamma_{1}^{2}+\gamma_{1}|\beta|+\gamma_{2}^{2}+2 \gamma_{2}+|\beta| \gamma_{2} / 2}{1-|\beta| / 2}\right)
\end{aligned}
$$

As follows. 
Proof. Define the Lyapunov function on $\Re^{2}$

$$
V\left(x_{1}, x_{2}\right)=x_{1}^{2}-\beta x_{1} x_{2}+x_{2}^{2}
$$

Applying Itô's formula to (77), we have

$$
\begin{aligned}
d V\left(x_{1}, x_{2}\right)= & {\left[\beta x_{2}^{2}+\beta x_{1}^{2}-\beta^{2} x_{1} x_{2}+\sigma_{1}^{2} x_{1}^{2}+\sigma_{2}^{2} x_{2}^{2}\right] d t } \\
& +\left[2 \sigma_{2} x_{2}^{2}+\left(2 \sigma_{1}-\beta \sigma_{2}\right) x_{1} x_{2}-\beta \sigma_{1} x_{1}^{2}\right] d W(t) \\
& +\left[\left(\gamma_{2}^{2}+2 \gamma_{2}\right) x_{2}^{2}+\left(2 \gamma_{1}-\gamma_{2} \beta\right) x_{1} x_{2}\right. \\
& \left.+\left(\gamma_{1}^{2}-\gamma_{1} \beta\right) x_{1}^{2}\right] d N(t) .
\end{aligned}
$$

Since

$$
\begin{gathered}
2 \sigma_{2} x_{2}^{2}+2 \sigma_{1} x_{1} x_{2} \leq\left(2 \sigma_{2}+\sigma_{1}\right)\left(x_{1}^{2}+x_{2}^{2}\right) \\
-\beta \sigma_{2} x_{1} x_{2}-\beta \sigma_{1} x_{1}^{2} \leq|\beta|\left(\sigma_{1}+\frac{\sigma_{2}}{2}\right)\left(x_{1}^{2}+x_{2}^{2}\right) \\
2 x_{1} x_{2}+\left(\gamma_{1}^{2}-\gamma_{1} \beta\right) x_{1}^{2} \leq\left(1+\gamma_{1}^{2}+\gamma_{1}|\beta|\right)\left(x_{1}^{2}+x_{2}^{2}\right) \\
\left(\gamma_{2}^{2}+2 \gamma_{2}\right) x_{2}^{2}-\beta \gamma_{2} x_{1} x_{2} \\
\leq\left(1+\gamma_{2}^{2}+2 \gamma_{2}+\frac{|\beta| \gamma_{2}}{2}\right)\left(x_{1}^{2}+x_{2}^{2}\right) \\
\left(1-\frac{|\beta|}{2}\right)\left(x_{1}^{2}+x_{2}^{2}\right) \leq V\left(x_{1}, x_{2}\right) \leq\left(1+\frac{|\beta|}{2}\right)\left(x_{1}^{2}+x_{2}^{2}\right) .
\end{gathered}
$$

From (78) and (79), we have

$$
\begin{aligned}
& d V\left(x_{1}, x_{2}\right) \\
& \leq\left[\beta+\frac{\max \left\{\sigma_{1}^{2}, \sigma_{2}^{2}\right\}}{1-|\beta| / 2}\right] V\left(x_{1}, x_{2}\right) d t \\
& +\left[\frac{\left(2 \sigma_{2}+\sigma_{1}\right)+|\beta|\left(\sigma_{1}+\sigma_{2} / 2\right)}{1-|\beta| / 2}\right] V\left(x_{1}, x_{2}\right) d W(t) \\
& +\left[\frac{2+\gamma_{1}^{2}+\gamma_{1}|\beta|+\gamma_{2}^{2}+2 \gamma_{2}+|\beta| \gamma_{2} / 2}{1-|\beta| / 2}\right] \\
& \quad \times V\left(x_{1}, x_{2}\right) d N(t),
\end{aligned}
$$

$$
\begin{aligned}
& d V\left(x_{1}, x_{2}\right) \\
& \geq\left[\beta+\frac{\max \left\{\sigma_{1}^{2}, \sigma_{2}^{2}\right\}}{1+(|\beta| / 2)}\right] V\left(x_{1}, x_{2}\right) d t \\
& \quad+\left[\frac{\left(2 \sigma_{2}+\sigma_{1}\right)+|\beta|\left(\sigma_{1}+\sigma_{2} / 2\right)}{1+|\beta| / 2}\right] \\
& \quad \times V\left(x_{1}, x_{2}\right) d W(t) \\
& \quad+\left[\frac{2+\gamma_{1}^{2}+\gamma_{1}|\beta|+\gamma_{2}^{2}+2 \gamma_{2}+|\beta| \gamma_{2} / 2}{1+|\beta| / 2}\right] \\
& \quad \times V\left(x_{1}, x_{2}\right) d N(t) .
\end{aligned}
$$

By Doléans-Dade's exponent formula, solving the corresponding linear differential equation, we have

$$
\begin{aligned}
V\left(x_{1}, x_{2}\right) \\
=\left\{\begin{array}{l}
\leq V\left(t_{0}\right) \exp \{a t+b W(t)+\log (1+c) N(t)\}, \\
\geq V\left(t_{0}\right) \exp \{k t+h W(t)+\log (1+r) N(t)\},
\end{array}\right.
\end{aligned}
$$

where

$$
\begin{gathered}
a=\left[\beta+\frac{\left.\max \left\{\sigma_{1}^{2}, \sigma_{2}^{2}\right\}\right]}{1-|\beta| / 2}\right] \\
-\frac{\left[\left(\left(2 \sigma_{2}+\sigma_{1}\right)-\beta\left(\sigma_{1}+\sigma_{2} / 2\right)\right) /(1-|\beta| / 2)\right]^{2}}{2}, \\
b=\frac{\left(2 \sigma_{2}+\sigma_{1}\right)-\beta\left(\sigma_{1}+\sigma_{2} / 2\right)}{1-|\beta| / 2}, \\
k=\frac{2+\gamma_{1}^{2}-\gamma_{1} \beta+\gamma_{2}^{2}+2 \gamma_{2}-\beta \gamma_{2} / 2}{1-|\beta| / 2}, \\
\left.\beta+\frac{\max \left\{\sigma_{1}^{2}, \sigma_{2}^{2}\right\}}{1+|\beta| / 2}\right] \\
-\frac{\left.\left[\left(2 \sigma_{2}+\sigma_{1}\right)-\beta\left(\sigma_{1}+\sigma_{2} / 2\right)\right) /(1+|\beta| / 2)\right]^{2}}{2}, \\
k=\frac{\left(2 \sigma_{2}+\sigma_{1}\right)-\beta\left(\sigma_{1}+\sigma_{2} / 2\right)}{1+|\beta| / 2}, \\
r=\frac{2+\gamma_{1}^{2}-\gamma_{1} \beta+\gamma_{2}^{2}+2 \gamma_{2}-\beta \gamma_{2} / 2}{1+|\beta| / 2} .
\end{gathered}
$$


Then, we can obtain

$$
\begin{aligned}
\lim _{t \rightarrow \infty} \frac{\log \left(V\left(x_{1}, x_{2}\right)\right)}{t} \\
=\left\{\begin{array}{l}
\leq \lim _{t \rightarrow \infty} \frac{\log (a t+b W(t)+\log (1+c) N(t))}{t} \\
=a+\lambda \log (1+c), \\
\geq \lim _{t \rightarrow \infty} \frac{\log (k t+h W(t)+\log (1+r) N(t))}{t} \\
=k+\lambda \log (1+r) .
\end{array}\right.
\end{aligned}
$$

Dividing by $t$ and letting $t \rightarrow \infty$, one obtains the claimed estimate on the Lyapunov exponents.

Theorem 10. Suppose the condition of Theorem 3 is satisfied and the coefficient in (3) is satisfied and $\alpha=-1$ and $\beta \in(-2,0)$ such that

$$
\begin{aligned}
\Lambda= & {\left[\beta+\frac{\max \left\{\sigma_{1}^{2}, \sigma_{2}^{2}\right\}}{1-|\beta| / 2}\right] } \\
& -\frac{\left[\left(\left(2 \sigma_{2}+\sigma_{1}\right)+|\beta|\left(\sigma_{1}+\sigma_{2} / 2\right)\right) /(1-|\beta| / 2)\right]^{2}}{2} \\
& +\lambda \log \left(1+\frac{2+\gamma_{1}^{2}+\gamma_{1}|\beta|+\gamma_{2}^{2}+2 \gamma_{2}+|\beta| \gamma_{2} / 2}{1-|\beta| / 2}\right)<0 .
\end{aligned}
$$

Then, the random dynamical system generated by the stochastic Rayleigh-van der Pol equation (3) possesses the unique tempered random attractor

$$
A(\omega)=\{0\},
$$

with domain of attractor $\mathscr{D}(A)$ containing the universe of $\operatorname{Cl}(\mathcal{U})$ given by

$$
\mathcal{U}=\left\{(D(\omega))_{\omega \in \Omega}: D(\omega) \subset \mathfrak{R}^{2} \text { is a tempered random set }\right\}
$$

where $\mathscr{C l}(\mathcal{U})$ is the complete universe of $\mathscr{U}$ under inclusion.

In particular, for any $x \in \mathfrak{R}^{2}$, the solutions $\varphi\left(t, \theta_{-t} \omega\right) x$ and $\varphi(t, \omega) x$ tend to zero exponentially fast as $t \rightarrow \infty$.

Proof. By Proposition 9 and assumption (91), the random dynamical system generated by (3) is stable. Let us define the nonnegative function on $\mathfrak{R}^{2}$ as follows:

$$
V\left(x_{1}, x_{2}\right)=-\beta x_{1}^{4}+x_{1}^{2}-\beta x_{1} x_{2}+x_{2}^{2} .
$$

Applying Itô's formula to (87), we can obtain

$$
\begin{aligned}
d V\left(x_{1}, x_{2}\right)=[ & \beta x_{2}^{2}+\beta x_{1}^{2}-\beta^{2} x_{1} x_{2}+\sigma_{1}^{2} x_{1}^{2} \\
& \left.+\sigma_{2}^{2} x_{2}^{2}+\beta x_{1} x_{2}^{3}-2 x_{2}^{4}-2 x_{1}^{2} x_{2}^{2}\right] d t \\
+ & {\left[2 \sigma_{2} x_{2}^{2}+\left(2 \sigma_{1}-\beta \sigma_{2}\right) x_{1} x_{2}-\beta \sigma_{1} x_{1}^{2}\right] d W(t) } \\
+ & {\left[\left(\gamma_{2}^{2}+2 \gamma_{2}\right) x_{2}^{2}+\left(2 \gamma_{1}-\gamma_{2} \beta\right) x_{1} x_{2}\right.} \\
& \left.+\left(\gamma_{1}^{2}-\gamma_{1} \beta\right) x_{1}^{2}\right] d N(t) .
\end{aligned}
$$

From (79) and (88), we can obtain

$$
\begin{aligned}
& d V\left(x_{1}, x_{2}\right) \\
& \leq\left\{\left[\beta+\frac{\max \left\{\sigma_{1}^{2}, \sigma_{2}^{2}\right\}}{1-|\beta| / 2}\right] V\left(x_{1}, x_{2}\right)+R(\beta)\right\} d t \\
& \quad+\left[\frac{\left(2 \sigma_{2}+\sigma_{1}\right)+|\beta|\left(\sigma_{1}+\sigma_{2} / 2\right)}{1-|\beta| / 2}\right] V\left(x_{1}, x_{2}\right) d W(t) \\
& \quad+\left[\frac{2+\gamma_{1}^{2}+\gamma_{1}|\beta|+\gamma_{2}^{2}+2 \gamma_{2}+|\beta| \gamma_{2} / 2}{1-|\beta| / 2}\right] \\
& \quad \times V\left(x_{1}, x_{2}\right) d N(t),
\end{aligned}
$$

where $R\left(x_{1}, x_{2}\right)=\beta x_{1} x_{2}^{3}-2 x_{2}^{4}-2 x_{1}^{2} x_{2}^{2}$ is uniformly bounded by a positive constant $R(\beta)$.

By Lemma 5, we can obtain that the random dynamical system generated by the stochastic differential equation

$$
\begin{aligned}
d X_{t} \leq & \left\{\left[\beta+\frac{\max \left\{\sigma_{1}^{2}, \sigma_{2}^{2}\right\}}{1-|\beta| / 2}\right] X_{t}+R(\beta)\right\} d t \\
& +\left[\frac{\left(2 \sigma_{2}+\sigma_{1}\right)+|\beta|\left(\sigma_{1}+\sigma_{2} / 2\right)}{1-|\beta| / 2}\right] X_{t} d W(t) \\
& +\left[\frac{2+\gamma_{1}^{2}+\gamma_{1}|\beta|+\gamma_{2}^{2}+2 \gamma_{2}+|\beta| \gamma_{2} / 2}{1-|\beta| / 2}\right] X_{t} d N(t),
\end{aligned}
$$

has the unique invariant measure $\delta_{0}$ which is the Dirac measure at 0 . From the proof of Theorem $6, A(\omega)=\{0\}=$ $V^{-}(\{0\})$ is an attractor attracting any set form $\mathcal{U}$.

In particular, we obtain that for any $x \in \mathfrak{R}^{2}, \varphi\left(t, \vartheta_{-t}, x\right)$ and $\varphi(t, \omega, x)$ tend to zero as $t \rightarrow \infty$ with an exponential speed larger than $\Lambda$.

$A(\omega)=A^{\mathfrak{R}^{2}}(\omega)$ is immediate from Theorem 6 and the definition of $\mathcal{U}$.

Theorem 11. Suppose the condition of Theorem 3 is satisfied and the coefficient in (3) is satisfied and $\alpha=-1$ and $\beta \in(0,2)$ such that

$$
\begin{aligned}
\Lambda= & {\left[\beta+\frac{\max \left\{\sigma_{1}^{2}, \sigma_{2}^{2}\right\}}{1+|\beta| / 2}\right] } \\
& -\frac{\left[\left(\left(2 \sigma_{2}+\sigma_{1}\right)+|\beta|\left(\sigma_{1}+\sigma_{2} / 2\right)\right) /(1+|\beta| / 2)\right]^{2}}{2} \\
& +\lambda \log \left(1+\frac{2+\gamma_{1}^{2}+\gamma_{1}|\beta|+\gamma_{2}^{2}+2 \gamma_{2}+|\beta| \gamma_{2} / 2}{1+|\beta| / 2}\right)
\end{aligned}
$$

$>0$. 
Then, the random dynamical system generated by the stochastic Rayleigh-van der Pol equations (1) possesses the unique tempered random attractor

$$
A(\omega)=\Re^{2} \backslash\{0\},
$$

with domain of attractor $\mathscr{D}(A)$ containing the universe of $\operatorname{Cl}(\mathcal{U})$ given by

$$
\begin{gathered}
\mathcal{U}=\left\{\text { all random } \operatorname{set}(D(\omega))_{\omega \in \Omega} \subset \mathfrak{R}^{2} \backslash\{0\}:\right. \\
\left.\sup _{x \in D(\omega)}\left(\|x\|+\frac{1}{\|x\|}\right) \text { is tempered }\right\} .
\end{gathered}
$$

$\mathcal{U}$ contains any random set "living" in an annulus surrounding zero whose outer boundary does not grow exponentially fast and whose inner boundary does not tend to zero exponentially fast.

Proof. It is the same as the proof in Theorem 10.

\section{Acknowledgments}

This research was supported by the National Natural Science Foundation of China (nos. 11201089 and 11271139) and by Guangxi Natural Science Foundation (nos. 2013GXNSFAA019014 and 2013GXNSFBA019016).

\section{References}

[1] D. Applebaum, Lévy Processes and Stochastic Calculus, vol. 116 of Cambridge Studies in Advanced Mathematics, Cambridge University Press, Cambridge, UK, 2nd edition, 2009.

[2] R. F. Bass, "Stochastic differential equations with jumps," Probability Surveys, vol. 1, pp. 1-19, 2004.

[3] C. W. Li, Z. Dong, and R. Situ, "Almost sure stability of linear stochastic differential equations with jumps," Probability Theory and Related Fields, vol. 123, no. 1, pp. 121-155, 2002.

[4] R. Situ, Theory of Stochastic Differential Equations with Jumps and Applications, Mathematical and Analytical Techniques with Applications to Engineering, Springer, Berlin, Germany, 2005.

[5] J. E. Figueroa-López, R. Gong, and C. Houdré, "Small-time expansions of the distributions, densities, and option prices of stochastic volatility models with Lévy jumps," Stochastic Processes and their Applications, vol. 122, no. 4, pp. 1808-1839, 2012.

[6] X. Sun and J. Duan, "Fokker-Planck equations for nonlinear dynamical systems driven by non-Gaussian Lévy processes," Journal of Mathematical Physics, vol. 53, no. 7, Article ID 072701, 10 pages, 2012.

[7] L. Arnold, Random Dynamical Systems, Springer Monographs in Mathematics, Springer, New York, NY, USA, 1998.

[8] Z. Huang, Q. Yang, and J. Cao, "Stochastic stability and bifurcation for the chronic state in Marchuk's model with noise," Applied Mathematical Modelling, vol. 35, no. 12, pp. 5842-5855, 2011.

[9] K. R. Schenk-Hoppé, "Random attractors-general properties, existence and applications to stochastic bifurcation theory," Discrete and Continuous Dynamical Systems, vol. 4, no. 1, pp. 99-130, 1998.
[10] J. Li and W. Xu, "Stochastic stabilization of first-passage failure of Rayleigh oscillator under Gaussian white-noise parametric excitations," Chaos, Solitons and Fractals, vol. 26, no. 5, pp. 15151521,2005

[11] Z. Lin, J. Liu, W. Zhang, and Y. Niu, "Stabilization of interconnected nonlinear stochastic Markovian jump systems via dissipativity approach," Automatica, vol. 47, no. 12, pp. 27962800, 2011.

[12] Y. Xie, "The random attractors of stochastic Duffing-van der Pol equations with jumps," Chinese Journal of Applied Probability and Statistics, vol. 26, no. 1, pp. 9-23, 2010.

[13] J. Touboul and G. Wainribc, "Bifurcations of stochastic differential equations with singular diffusion coefficients," http://arxiv.org/abs/1205.0172.

[14] H. Fink and C. Klüppelberg, "Fractional Lévy-driven OrnsteinUhlenbeck processes and stochastic differential equations," Bernoulli, vol. 17, no. 1, pp. 484-506, 2011.

[15] C. W. Li and G. L. Blankenship, "Almost sure stability of linear stochastic systems with Poisson process coefficients," SIAM Journal on Applied Mathematics, vol. 46, no. 5, pp. 875-911, 1986.

[16] S. Rong, "On solutions of backward stochastic differential equations with jumps and applications," Stochastic Processes and their Applications, vol. 66, no. 2, pp. 209-236, 1997. 


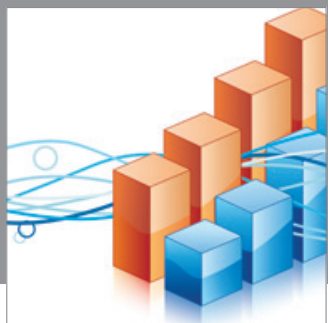

Advances in

Operations Research

mansans

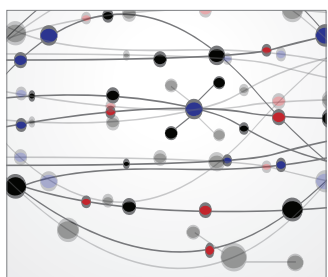

The Scientific World Journal
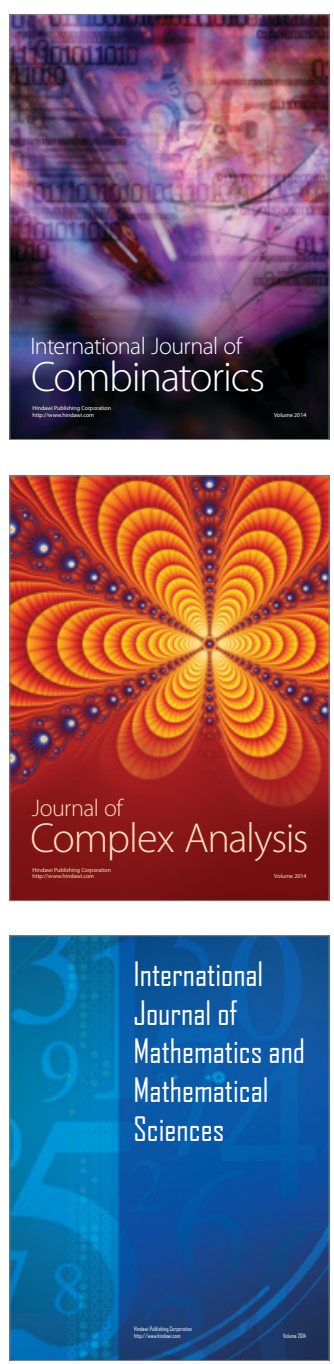
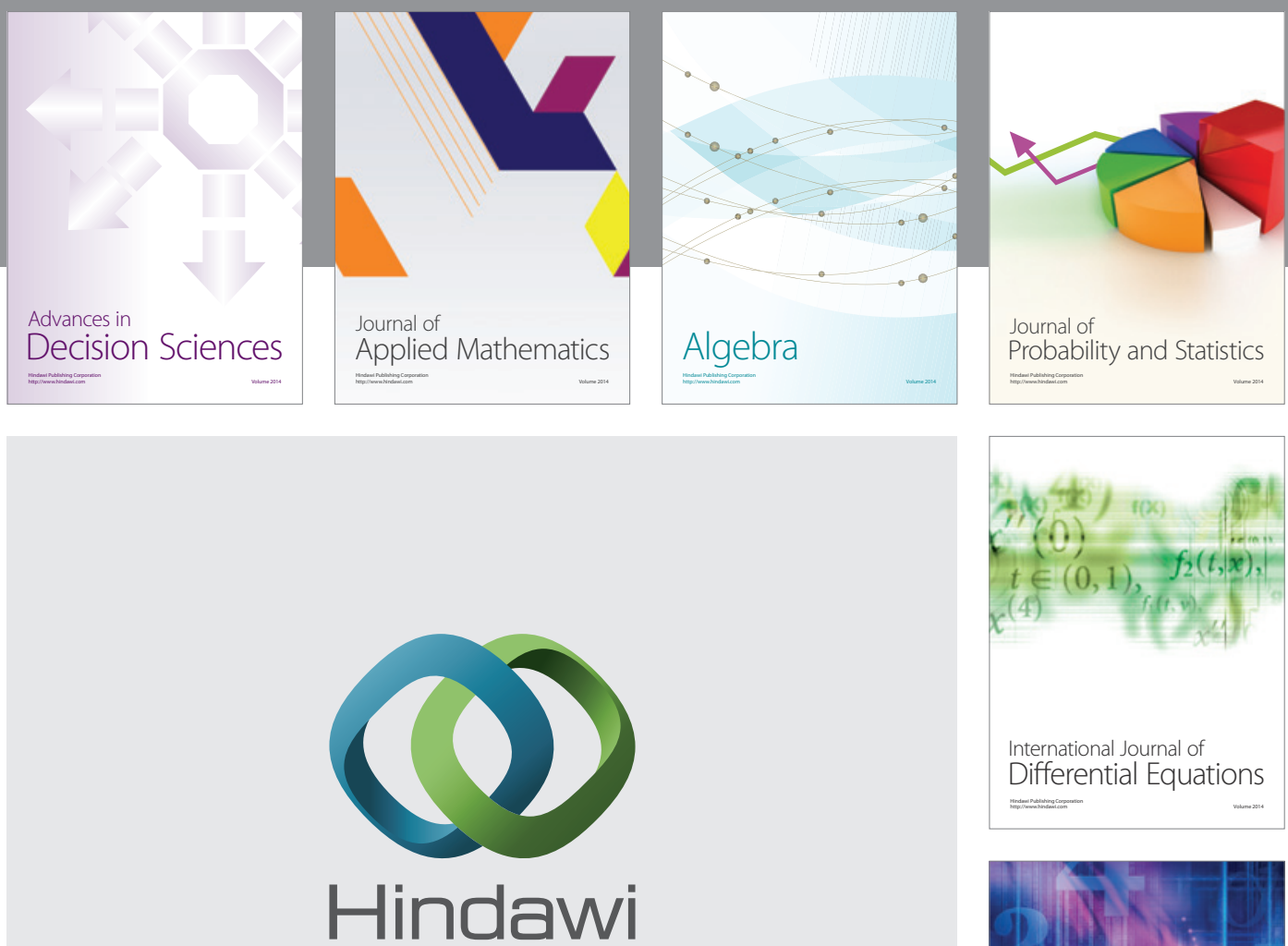

Submit your manuscripts at http://www.hindawi.com
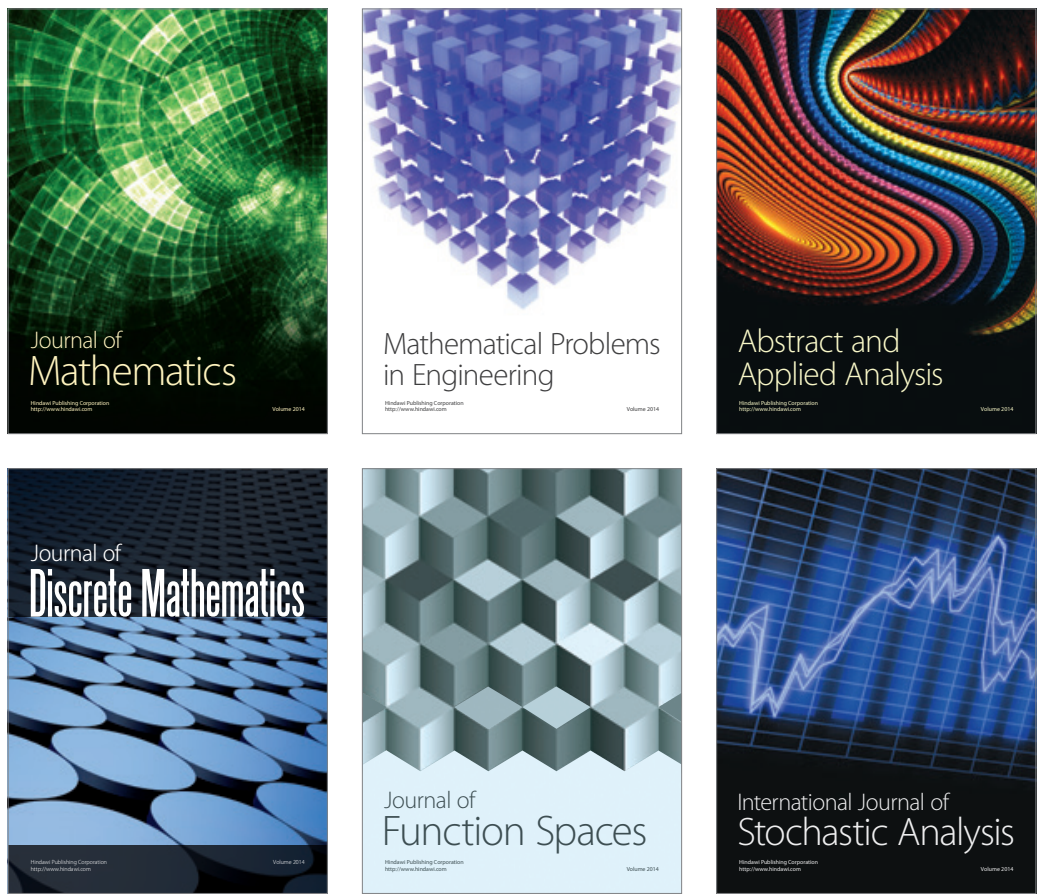

Journal of

Function Spaces

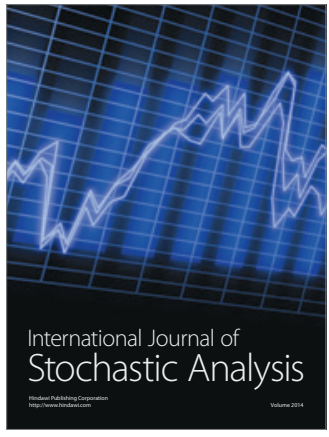

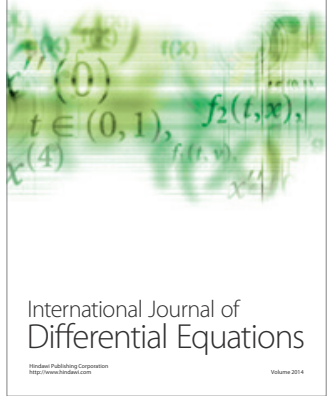
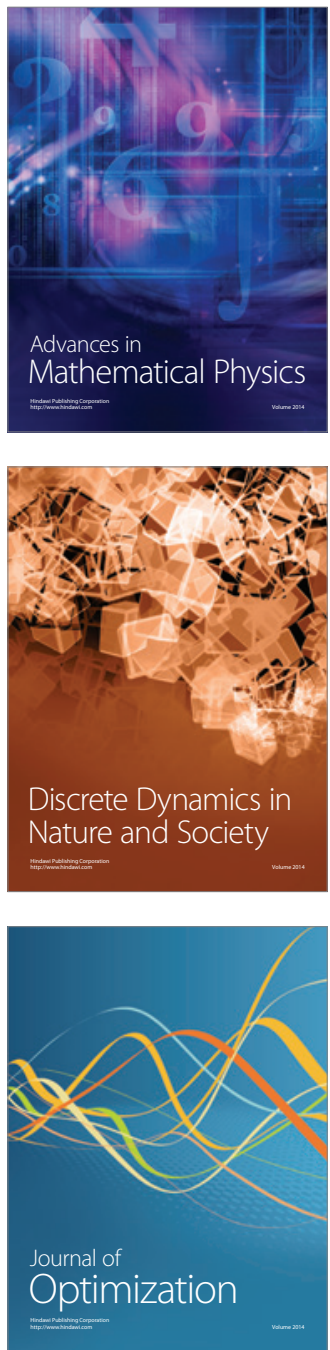